\title{
Dendritic and spinal alterations of neurons from Edinger-Westphal nucleus in Alzheimer's disease
}

\author{
Ioannis Asterios Mavroudis ${ }^{1}$, Marina George Manani ${ }^{1}$, Foivos Petrides ${ }^{1}$, Constantina Petsoglou ${ }^{2}$, Samuel N. Njau ${ }^{2}$, \\ Vasiliki G. Costa ${ }^{1,3}$, Stavros J. Baloyannis ${ }^{1,3}$ \\ ${ }^{1}$ Laboratory of Neuropathology and Electron Microscopy, $1^{\text {st }}$ Department of Neurology, Aristotelian University of Thessaloniki, ${ }^{2}$ Labora- \\ tory of Forensic Medicine and Toxicology, Aristotelian University of Thessaloniki, ${ }^{3}$ nnstitute for Alzheimer's disease research, Heraklion \\ Langada, Greece
}

\begin{abstract}
Alzheimer's disease $(A D)$ is a heterogeneous neurodegenerative disorder, causing a progressive decline of intellectual faculties, impairment of behavior and social performance, and impairment of speech eloquence, associated with various neurological manifestations based on a variable neuropathological background. Edinger-Westphal nucleus is a selective target of Alzheimer pathology early in the course of the disease. We attempted to determine the morphological alterations of the dendrites and the dendritic spines in Edinger-Westphal nucleus of 7 cases that fulfilled the diagnostic criteria for Alzheimer's disease. For the histological study, we applied (a) routine neuropathological techniques and (b) rapid Golgi method. We proceeded to 3D neuronal reconstruction for the estimation of dendritic and spinal changes in Alzheimer's disease. The morphological and morphometric analysis revealed a substantial neuronal loss and synaptic alterations in Edinger-Westphal nucleus in all the cases of Alzheimer's disease. Distal dendritic branches are prominently affected. The neuronal loss and alteration of the spines in Edinger-Westphal nucleus in Alzheimer's disease may be related to the exaggerated pupillary reaction to cholinergic antagonists. Furthermore, the vulnerability of distal branches to Alzheimer's disease might be related to neuroplasticity impairment.
\end{abstract}

Key words: Alzheimer's disease, Golgi method, 3D neuronal reconstruction, Edinger-Westphal nucleus.

\section{Introduction}

Alzheimer's disease (AD) is the primary cause of dementia affecting, according to recent studies, as much as $6-8 \%$ of people over the age of 65 years and nearly $30 \%$ of people older than 85 years [6,31]. It is physically characterized by a progressive decline of intellectual faculties, loss of professional skills, and variable deficits in perceptual, visual and auditory functions [27,32]. Macroscopically, cerebral atro- phy is easily recognized in the majority of cases of Alzheimer's disease, being the most obvious in the temporal and parietal lobes [1,14]. Light microscopy reveals senile plaques and neurofibrillary tangles in the entorhinal cortex and hippocampus before spreading in other brain areas such as the acoustic and visual cortex, frontal cortex, and cerebellum $[5,18,19,30,35]$. 
Alzheimer's disease is found to be associated with reduced levels of acetylcholine, noradrenaline, serotonin, somatostatin, and corticotrophin-releasing factors, whereas the levels of glutamate increase [7]. The cognitive deficits are attributable to the degeneration of cholinergic neurons in the basal-forebrain-cholinergic system that projects to the neocortex, hippocampus, and other brain areas [7,11].

The nucleus of Edinger-Westphal is the most rostral of the parasympathetic nuclei in the brainstem and supplies parasympathetic fibers to the eye [21].

The nucleus is located posteriorly to the main CNIII motor nucleus (oculomotor nucleus) and anterolaterally to the cerebral aqueduct in the rostral midbrain at the level of the superior colliculus [3].

Edinger-Westphal (EW) nucleus is a selective target of Alzheimer pathology early in the course of the disease [29]. Scinto et al. showed severe focal pathology of the EW nucleus in AD. The same authors noticed that patients who suffer from $A D$ show an exaggerated pupillary reaction to the cholinergic antagonist tropicamide and in 2001, noticed a significant selective neuronal loss in the EW nucleus, whereas the number of neurons in the somatic portion of the nucleus of the third cranial nerve remained intact $[28,29]$. They showed that although the somatic portion of the third cranial nerve nuclei did not show any signs of pathology, the EW nucleus contained plaques and tangles in $\mathrm{AD}$ patients.

In previous studies of ours using the Golgi method and 3D neuronal reconstruction techniques, severe dendritic and spinal alterations were seen in Alzheimer's disease and normal aged brains [18-20].

In the present study, we intend to describe the morphometric and morphological changes of the neurons of the Edinger-Westphal nucleus in Alzheimer's disease in comparison to normal ageing and to investigate if except for the neuronal loss, senile plaques deposition and neurofibrillary degeneration, the EW nucleus is characterized by dendritic and spinal alterations in Alzheimer's disease.

\section{Material and methods \\ Subjects}

Tissue samples were obtained from 8 neurologically normal individuals post-mortem, and 7 with Alzheimer's disease, all of them aged between 67 and 86 years (mean $77.4 \pm 5.1$ ). The average autolysis time for all subjects was $12 \pm 4$ hours.
All brains, after their excision from the skull were immersed in $10 \%$ neutral buffered formalin for at least 25 days.

All possible information on each subject, concerning their previous physical and illness history, was obtained from autopsy reports as well as medical records. All the brains were examined by an independent neuropathologist for gross and microscopic signs of pathology. The brains did not exhibit trauma, edema or chronic illness. No neurofibrillary tangles or senile plaques were observed in normal controls, whereas AD brains were classified as stage V/VI according to Braak and Braak classification and CERAD score indicative of the disease. Histological criteria for the diagnosis of $A D$ were those outlined by the National Institutes of Health/American Association of Retired Persons (NIH/AARP) Research Workshop on the Diagnosis of Alzheimer's Disease. All cases fulfilled the histological criteria for AD [18].

\section{Tissue selection and processing}

A tissue block from the Edinger-Westphal nucleus was excised. The EW nucleus was identified according to Scinto et al.'s studies [28,29]. The tissue blocks were coded in order to prevent experimental bias and were used for silver techniques [18-20], Congo red method [2], Bielschowsky staining [16], Gallyas technique [33] and Nissl staining [24].

\section{Cell selection criteria}

Neurons examined for quantitative alterations met the criteria set forth by Jacobs et al. [15] that request uniform staining of neuronal processes, absence of precipitated debris, good contrast between cells and background and relatively uniform tissue thickness.

\section{Golgi method}

For silver impregnation, the specimens were immediately immersed in a dilution of potassium dichromate $(7 \mathrm{~g}$ of potassium dichromate and $20 \mathrm{~mL}$ of formaldehyde solution $37 \%$ in $300 \mathrm{~mL}$ of tap water) at room temperature. They remained in that solution for one week, and then they were immersed in an aqueous solution of $1 \%$ silver nitrate where they remained for one more week at a temperature of $15^{\circ} \mathrm{C}$ in a photoprotected environment.

After fixation, the specimens were embedded in low-melting-point paraffin and cut with a slicing microtome in thick sections at a range of $120 \mu \mathrm{m}$ 
and after rapid differentiation they were covered with entellan.

\section{Nissl staining and Congo red technique}

Adjacent sections were cut in a range of $20 \mu \mathrm{m}$ and used for Bielschowsky staining [16], Gallyas technique [33], Nissl staining [24] and Congo red method [2] in order to evaluate the neuronal population, the neurofibrillary degeneration and the deposition of senile and amyloid plaques.

\section{Neuronal tracing and dendritic quantification}

For each one of the 15 brains, 10 neuronal cells from each area were selected. For every cell, we took a 10 -second video at a magnification of $400 \times$ while the microscope table was moving at the standard velocity of $20 \mu \mathrm{m} / \mathrm{sec}$. Afterwards, the videos were analyzed in digital image sequences of 200 serial pictures, which were ultimately imported in Neuromantic application to trace the cells, quantifying them along $x$-, $y$ - and $z$-coordinates.

Each one of the selected cells was traced using the Neuromantic application. The neuronal tracing started with the cell soma and moved onto the basilar dendrites and the apical shaft.

Dendritic trees were quantitatively evaluated in a centrifugal manner for apical dendrites and basal dendrites according to Uylings et al. [34]. Dendrites arising from the cell soma are considered first-order segments, up to their first symmetrical bifurcation. Dendritic branches arising from first-order segments are considered second-order segments, in turn, up to their symmetrical bifurcation into third-order segments, and so on. When asymmetric branching is met during the neuronal tracing, the offspring dendritic branch, recognized by a qualitatively thinner diameter, is classified as a next-order branch, whereas the parent dendrite would retain its order level past the branching point [11].

\section{Dendritic measures and Sholl analysis}

The parameters measured were soma size, total dendritic length, cell contraction, dendritic field asymmetry, total number of dendritic segments and bifurcations, as well as the length and number of dendritic segments per order. Furthermore, the tracing was quantitatively analyzed with Image J program based on Sholl's [28] method of concentric circles. Concentric circles were drawn, at intervals of $15 \mu \mathrm{m}$ centered on the cell bodies, and dendritic intersections within each circle were counted.

\section{Spine counts}

Spine counts were carried out at 360 pictures, which were taken with an AxioCam HR, at the standard magnification of 1000x, on an Axiostar Plus photomicroscope. Visible spines were counted on three segments of the dendritic field. The first segment, 20-30 $\mu \mathrm{m}$ in length, was located on the first-order dendrite, the second segment, 20-30 $\mu \mathrm{m}$ in length, on second-order dendrite and the third one, 40-50 $\mu \mathrm{m}$, along the tertiary dendrite.

\section{Neuronal density}

For the estimation of neuronal density in each area, we used a modified semi-automatic optical dissector method provided by the Image I software. For each one of the brains, after defining the region of interest at a size of $150 \times 150 \mu \mathrm{m}$, we recorded a 3 -second video at a magnification of $400 \times$ while the microscope table was moving at the standard velocity of $20 \mu \mathrm{m} / \mathrm{sec}$. Afterwards, the videos were analyzed in digital image sequences of 50 serial pictures, which were converted to binary ones. Then, using the "cell counter" function in Image J, the neurons in each given ROI were counted.

\section{Statistical analysis}

Statistical analysis was based on the Student's test on the basis of 360 cells in SPSS v.17.0. Significance was taken as $p<0.05$. To ensure that autolysis time did not affect dendritic measurements, two-tailed Pearson product correlations were performed between all dependent measures and autolysis time.

\section{Results}

Golgi impregnation technique revealed a significant loss of distal dendritic branches and an overall restriction of the dendritic field in the AD brains. Tortuous branches and varicosities were also observed.

\section{Dendritic changes}

The total length of the dendritic field was significantly decreased in AD specimens. A severe loss of distal dendritic branches (quaternary), decrease in 
the total number of branches and bifurcations were also noticed. The branching ratio was also grossly affected.

Sholl analysis revealed a restriction of the dendritic field due to the loss of distal branches, although the proximal ones remained relatively intact (Fig. 1).

Neurons from the somatic portion of the third cranial nerve nuclei retained high spinal density and did not show statistically significant alterations in any of the morphometric parameters.

\section{A}

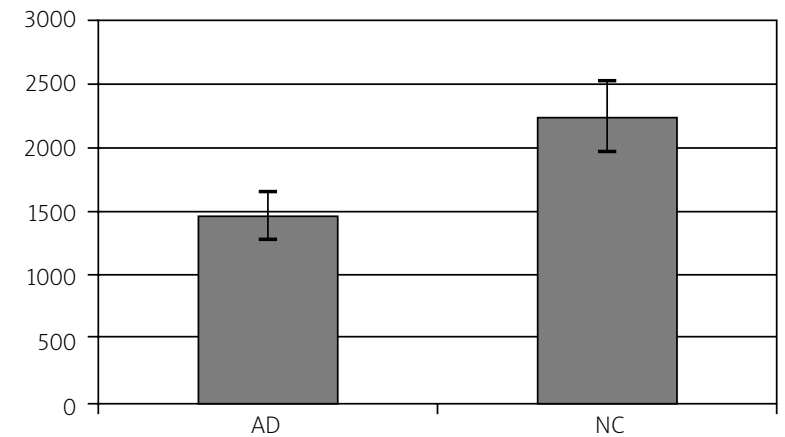

C

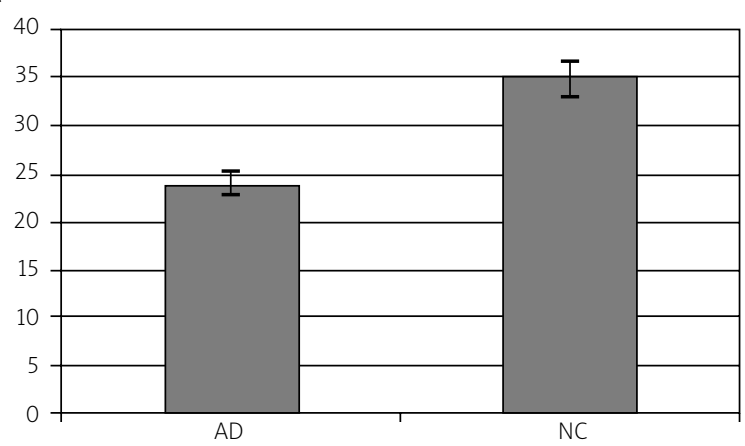

E

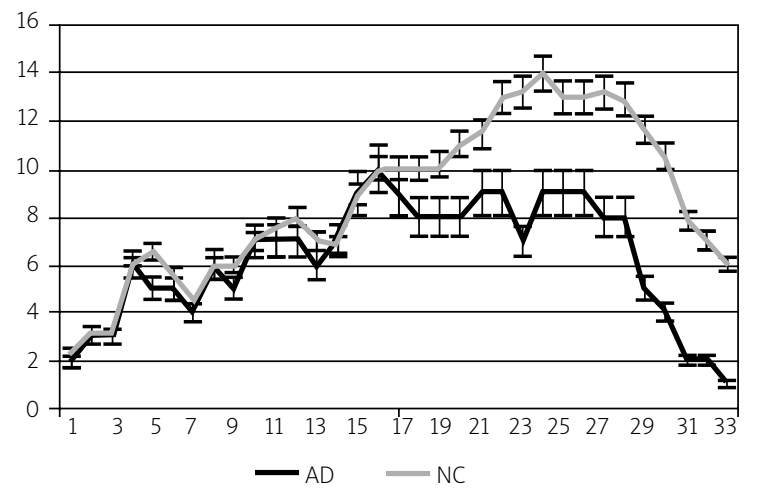

\section{Dendritic spines}

Spinal density was also significantly decreased in AD. Except for spinal loss, several branched, mushroom and claw ending spines were seen in the aged brains.

\section{Neuronal density}

Neuronal density in the Edinger-Westphal nuclei of $A D$ brains was severely decreased, whereas in the

\section{B}

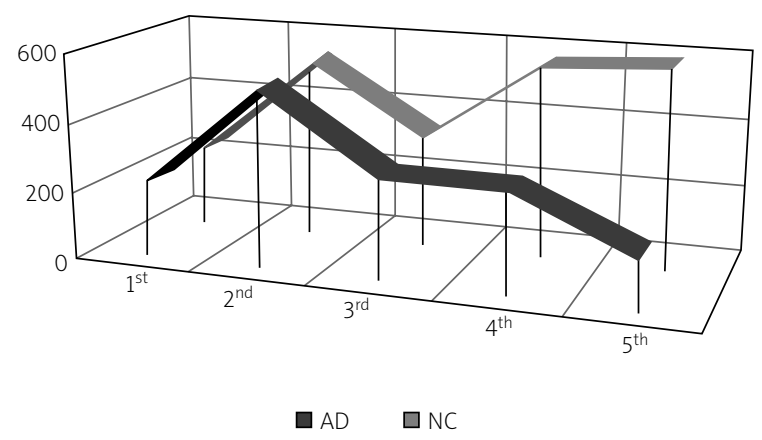

D

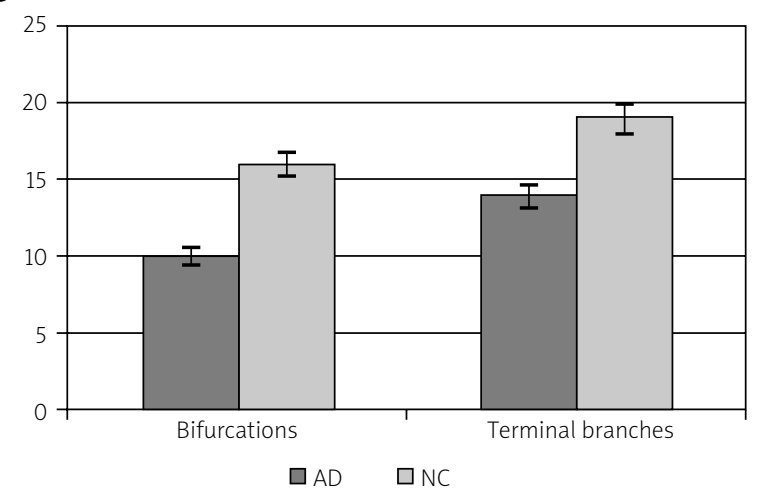

F

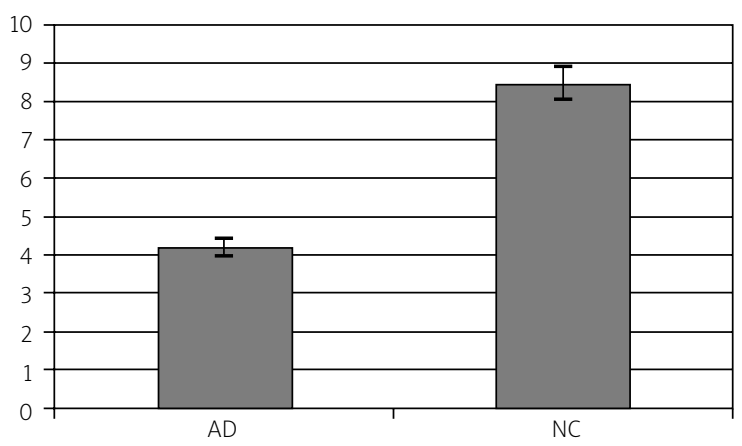

Fig. 1. A) Total dendritic length per neuron, B) dendritic length per branching order, C) total dendritic branches per neuron, D) number of bifurcations and terminal branches, E) Sholl analysis, and F) spine density in Edinger-Westphal neurons in Alzheimer's disease (AD) and in normal controls (NC). 
somatic portion of the oculomotor nuclei, no significant difference in the neuronal density was seen (Fig. 2).

\section{Senile plaques and neurofibrillary degeneration}

Gallyas technique revealed neurofibrillary degeneration in the majority of the EW neurons.

Two types of plaque-like deposits were mainly encountered in AD EW nuclei. Typical and compact amyloid cored plaques, were mainly observed, while diffuse plaques were seen outside the EW nuclei.

\section{Discussion}

Early studies made in the mid-1970s were the first one to describe substantial neocortical deficits in the enzyme responsible for the synthesis of acetylcholine, choline acetyltransferase $[4,8,26]$, while subsequent discoveries of reduced choline uptake, [28] acetylcholine release [23] and loss of cholinergic perikarya from the nucleus basalis of Meynert [35] confirmed the cholinergic deficit, leading to the cholinergic hypothesis of Alzheimer's disease.

On the other hand, biochemical studies showed a significant reduction of presynaptic markers of the cholinergic system early in the course of the disease [12].

From the neuropathologic point of view, Alzheimer's disease is characterized by neuronal loss, as well as dendritic and synaptic pathology in hippocampus and circumscribed regions of the neocor-

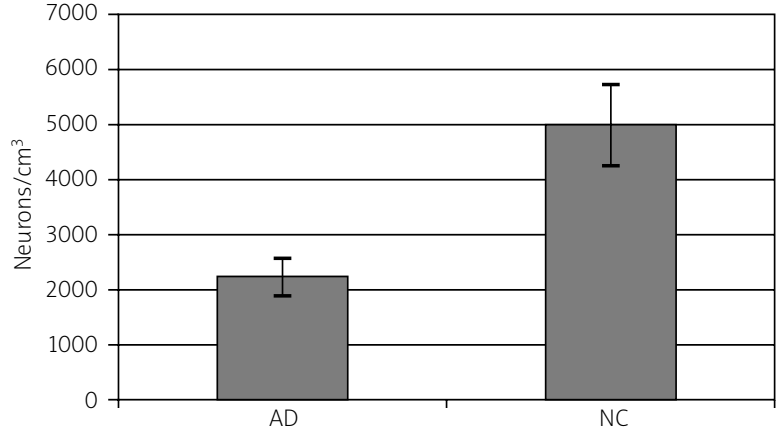

Fig. 2. Neuronal density (neurons $/ \mathrm{cm}^{3}$ ) in the Edinger-Westphal nucleus in Alzheimer's disease and normal controls.

tex $[9,17,19]$. Neurotransmitter-specific subcortical nuclei that project to the cortex are also affected by the implicated neurodegenerative processes, including the cholinergic nucleus basalis of Meynert and medial septum, the serotonergic raphe nuclei, and the noradrenergic locus coeruleus.

The nucleus of Edinger-Westphal is a selective target of Alzheimer pathology even in the early stages of the disease $[28,29]$.

Scinto et al. (1999) showed that the EW nucleus alone contained plaques and tangles in AD patients as well as a severe and selective neuronal loss, thus sparing the somatic portion of the third cranial nerve nucleus [29]. They addressed these findings congruent to the exaggerated pupillary reaction to
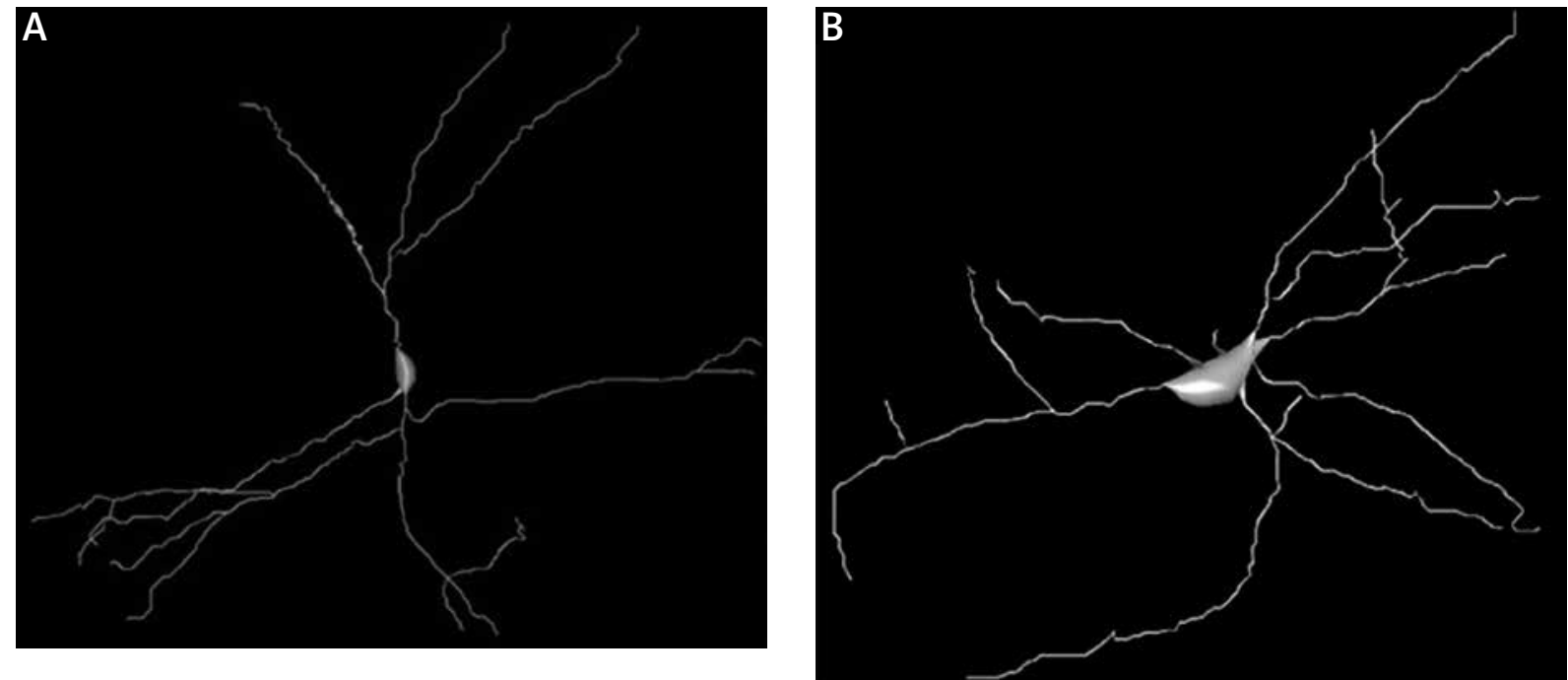

Fig. 3. Three dimensional reconstructions from a fusiform small soma sized neuron $(A)$ and a multipolar large soma sized (B) neuron of the Edinger-Westphal nucleus. 

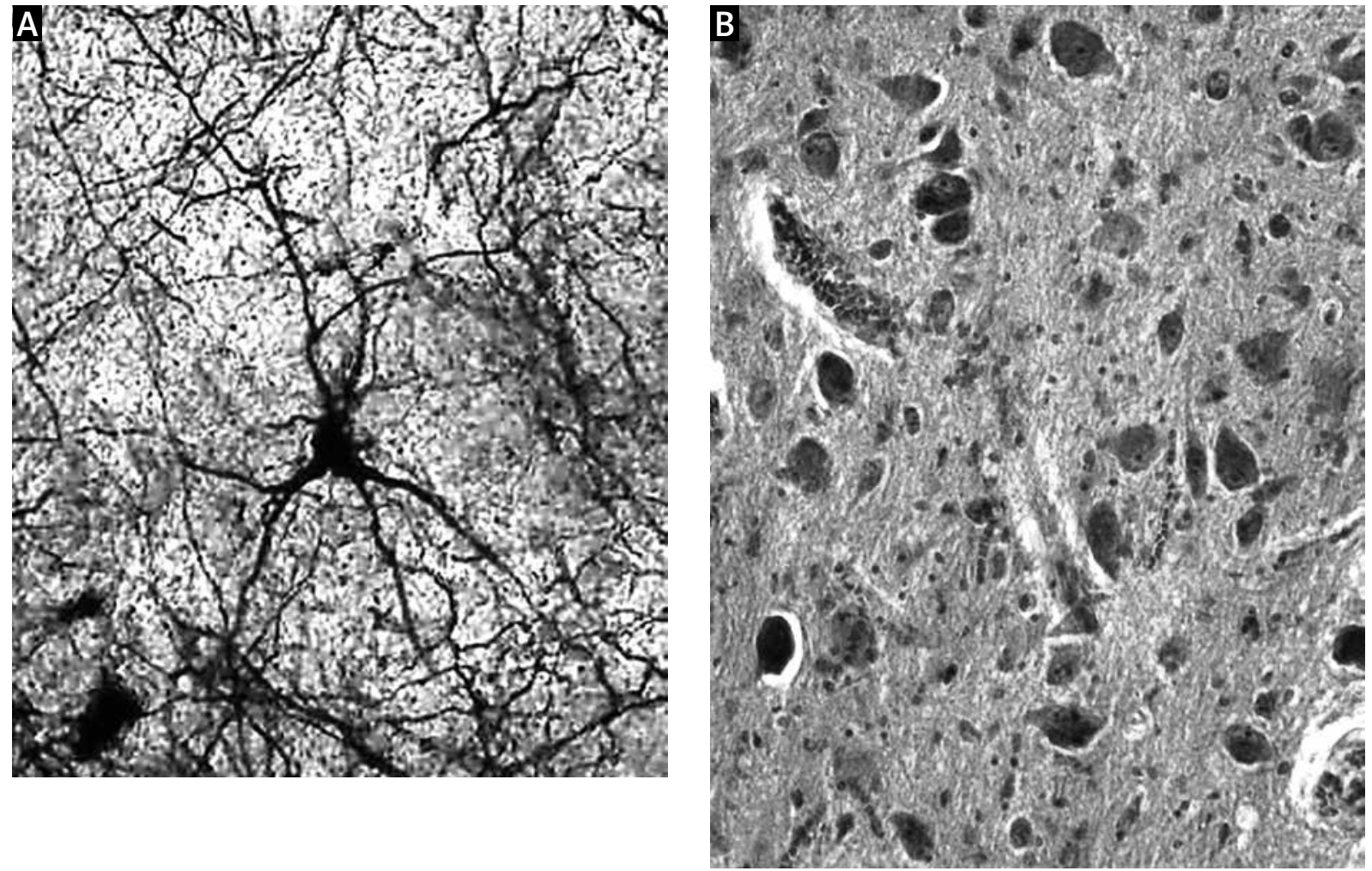

Fig. 4. A) Golgi stained neuron from the Edinger-Westphal nucleus from an AD brain, exhibiting a marked loss of dendrites, magnification 100×, B) Nissl stained neurons, astrocytes and microglial cells in the EdingerWestphal nucleus from an AD brain, magnification 400x.

the cholinergic antagonist tropicamide seen in the disease.

Morawski et al. (2010) showed a high expression of Glutaminyl cyclase in the cholinergic nuclei of Meynert and Edinger-Westphal, related to high vulnerability of well-defined subcortical neuronal populations and their cortical projections in AD [22].

In the present study we encountered severe dendritic and spinal pathology in the EW nucleus, in early AD cases using silver impregnation methods. A substantial loss of distal dendritic branches, significant reduction of the total dendritic length per neuron, and severe decrease in dendritic spines were seen. Neurons from the somatic portion of the oculomotor nerve nuclei remained relatively intact in comparison to the control brains. Furthermore Bielschowsky and Gallyas methods revealed a high concentration of senile plaques and neurofibrillary tangles, and Nissl method showed a severe neuronal loss confirming the findings by Scinto et al. [28,29].

The present study is innovative in terms of depiction of dendritic changes of the neurons of EW nucle- us, in relation to neurofibrillary degeneration and senile plaques deposition. Dendritic and spinal loss leads to a severe decrease in synaptic contacts. As a result, that should be related to the exaggerated pupillary reaction to cholinergic antagonists in AD. Furthermore, the present findings corroborate the hypothesis of cholinergic subcortical areas' vulnerability in AD.

An intriguing result of the present study is the prominent degeneration of the more distal branches of EW nucleus' neurons. From previous assays on similar topics, it is now acknowledged that distal branches are linked in the majority with neuronal plasticity and vice versa, therefore playing an important role in the process of learning and neuronal adaptation. On the other hand, neuronal plasticity is grossly affected by the accumulation of NFTs and is related to cholesterol metabolism, and therefore, at least ultimately, APP metabolism [13,19].

NFTs seem to clog dendritic branches leading to amputation of their more distal parts. As a result, they lead to both the severe loss of synapses and 
interruption of neuronal plasticity $[18,19]$. The fact that NFTs, a prominent feature of the AD brain, are seen in the same portions of the CNIII and EW nucleus provides a possible explanation of this result [19].

Moreover, ApoE is a major factor in the pathogenesis of $A D$, by means of cholesterol transfer and APP metabolism, leading also to the neuroplasticity dysfunction [13]. Therefore, it could mean that the neuroplasticity dysfunction causes the distal and synaptic loss found, or the other way around.

Additionally, in the present study, there was a significant change in the morphology of the spines, besides the loss in their number. Whereas in normal controls, the majority of spines were of the long-neck type, in $A D$ patients, about $80 \%$ of them were short-stubby ones, added the fact that giant dystrophic spines were also noticed. The morphology and structural plasticity of spines is related in a great amount to F-actin polymerization, which in turn, is thought to be a standard stage in NFT-linked degeneration. In conclusion, giant spines are usually a feature of increased oxidative stress, another alleged contributor of AD pathogenesis.

Summarizing the results, the present study, showing a loss of distal branches and alterations in spinal morphology and number that is coherent to the loss of synaptic contacts, comes in agreement with the disturbance of the neuronal function, thus providing an additional possible explanation for the pupillary reaction's particularities in AD.

\section{Disclosure}

Authors report no conflict of interest.

\section{References}

1. Baloyannis SJ (ed.). Neuropathology of Dementia. Thessaloniki, 1993.

2. Beauquis J, Pavía P, Pomilio C, Vinuesa A, Podlutskaya N, Galvan V, Saravia F. Environmental enrichment prevents astroglial pathological changes in the hippocampus of APP transgenic mice, model of Alzheimer's disease. Exp Neurol 2013; 239: 28-37.

3. Blokland A. Acetylcholine: a neurotransmitter for learning and memory? Brain Res Brain Res Rev 1995; 21: 285-300.

4. Bowen DM, Smith CB, White P, Davison AN. Neurotransmitter related enzymes and indices of hypoxia in senile dementia and other abiotrophies. Brain 1976; 99: 459-496.

5. Braak H, Braak E. Neuropathological staging of Alzheimer-related changes. Acta Neuropathol 1991; 82: 239-259.

6. Byrne GJ. Treatment of cognitive impairment in Alzheimer's disease. Aust J Hosp Pharm 1998; 28: 261-266.
7. Cutler NR, Sramek JJ. The role of bridging studies in the development of cholinesterase inhibitors for Alzheimer's disease. CNS Drugs 1998; 10: 355-364.

8. Davies P, Maloney AJF. Selective loss of central cholinergic neurons in Alzheimer's disease. Lancet 1976; 2: 1403.

9. De Kosky ST, Sche VSW, Styren SD. Structural correlates of cognition in dementia: quantification and assessment of synapse change. Neurodegeneration 1996; 5: 417-421.

10. Duan H, Wearne SL, Rocher AB, Macedo A, Morrison JH, Hof PR. Age-related dendritic and spine changes in corticocortically projecting neurons in macaque monkeys. Cereb Cortex 2003; 13: 950-961.

11. Francis PT, Palmer MA, Snape M, Wilcock GK. The cholinergic hypothesis of Alzheimer's disease: a review of progress. J Neurol Neurosurg Psychiatry 1999; 66: 137-147.

12. Francis PT, Sims NR, Procter AW, et al. Cortical pyramidal neuron loss may cause glutamatergic hypoactivity and cognitive impairment in Alzheimer's disease: investigative and therapeutic perspectives. I Neurochem 1993; 60: 1589-1604.

13. Hatzifilippou E, Banaki T, Traka M, Koutsouraki E, Costa V, Baloyannis SJ. Apolipoprotein E phenotype in demented patients in Greek population. Int I Neurosci 2008; 118: 163-172.

14. Hubbard BM, Anderson JM. A quantitative study of cerebral atrophy in old age and senile dementia. J Neurol Sci 1981; 50: 135-145.

15. Jacobs B, Driscoll L, Schall M. Life-span dendritic and spine changes in areas 10 and 18 of human cortex: A quantitative Golgi study. J Comp Neurol 1997; 386: 661-680.

16. Litchfield S, Nagy Z. New temperature modification makes the Bielschowsky silver stain reproducible. Acta Neuropathol 2001; 101: 17-21.

17. Mann DMA. Pyramidal nerve cell loss in Alzheimer's disease. Neurodegeneration 1996; 5: 423-429.

18. Mavroudis IA, Fotiou DF, Adipepe LF, Manani MG, Njau SD, Psaroulis D, Costa VG, Baloyannis SJ. Morphological changes of the human purkinje cells and deposition of neuritic plaques and neurofibrillary tangles on the cerebellar cortex of Alzheimer's disease. Am J Alzheimers Dis Other Demen 2010; 25: 585-591.

19. Mavroudis IA, Fotiou DF, Manani MG, Njaou SN, Frangou D, Costa VG, Baloyannis SJ. Dendritic and spinal pathology in Alzheimer's disease: A Golgi study in pathology. Int I Neurosci 2011; 121: 347-354.

20. Mavroudis I, Petrides F, Manani M, Theocharides C, Ciobica A, Padurariu M, Kiourexidou M, Njau S, Costa V, Baloyannis S. Dendritic and spinal alterations of the spiny stellate cells of the human visual cortex during normal aging. Folia Neuropathol 2012; 50: 261-269.

21. May PJ, Reiner AJ, Ryabinin AE. Comparison of the distributions of urocortin containing and cholinergic neurons in the perioculomotor midbrain of the cat and macaque. J Comp Neurol 2008; 507: 1300-1316.

22. Morawski M, Hartlage-Rübsamen M, Jäger C, Waniek A, Schilling S, Schwab C, McGeer PL, Arendt T, Demuth HU, Rossner S. Distinct glutaminyl cyclase expression in Edinger-Westphal nucleus, locus coeruleus and nucleus basalis Meynert contributes to pGlu-Abeta pathology in Alzheimer's disease. Acta Neuropathol 2010; 120: 195-207. 
23. Nilsson L, Nordberg A, Hardy JA, Wester P, Winblad B. Physostigmine restores $3 \mathrm{H}$-acetylcholine efflux from Alzheimer brain slices to normal level. J Neural Transm 1986; 67: 275-285.

24. Padurariu M, Ciobica A, Mavroudis I, Fotiou D, Baloyannis S. Hippocampal neuronal loss in the CA1 and CA3 areas of Alzheimer's disease patients. Psychiatr Danub 2012; 24: 152-158.

25. Perry E, Walker M, Grace J, Perry R. Acetylcholine in mind: a neurotransmitter correlate of consciousness? Trends Neurosci 1999; 22: 273-280.

26. Perry EK, Gibson PH, Blessed G, et al. Neurotransmitter enzyme abnormalities in senile dementia. Choline acetyltransferase and glutamic acid decarboxlyase activities in necropsy brain tissue. J Neurol Sci 1977; 34: 247-265.

27. Perry RJ, Watson P, Hodges JR. The nature and staging of attention dysfunction in early (minimal and mild) Alzheimer's disease: Relationship to episodic and semantic memory impairment. Neuropsychologica 2000; 38: 252-271.

28. Scinto LF, Frosch M, Wu CK, Daffner KR, Gedi N, Geula C. Selective cell loss in Edinger-Westphal in asymptomatic elders and Alzheimer's patients. Neurobiol Aging 2001; 22: 729-736.

29. Scinto LF, Wu CK, Firla KM, Daffner KR, Saroff D, Geula C. Foca pathology in the Edinger-Westphal nucleus explains pupillary hypersensitivity in Alzheimer's disease. Acta Neuropathol 1999; 97: 557-564.

30. Sholl DA. Dendritic organization in the neurons of the visual and motor cortices of the cat. J Anat 1953; 87: 387-406.

31. Small GW, Rabins PV, Barry PP, et al. Diagnosis and treatment of Alzheimer disease and related disorders. Consensus statement of the American Association for Geriatric Psychiatry, the Alzheimer's Association, and the American Geriatrics Society. JAMA 1997; 278: 1363-1371.

32. Terry RD, Katzman R. Senile dementia of Alzheimer's type. Ann Neurol 1983; 14: 497-506.

33. Tsamis K, Mytilinaios D, Njau SN, Psaroulis D, Mavroudis J, Costa V, Baloyannis SJ. The combination of silver techniques for studying the pathology of Alzheimer's disease. Int I Neurosci 2008; 118: 257-266.

34. Uylings HBM, Van Eden CG, Parnavelas JG, Kalsbeek A. The prenatal and postnatal development of rat cerebral cortex. In: Kolb E, Tees RC (eds). The cerebral cortex of the rat. MIT Press, Cambridge 1990; pp. 35-76.

35. Whitehouse PJ, Price DL, Struble RG, Clark AW, Coyle JT, Delon MR. Alzheimer's disease and senile dementia: loss of neurons in basal forebrain. Science 1982; 215: 1237-1239.

36. Xu Y, Jack CR Jr, O’Brien PC, Kokmen E, Smith EG, Ivnik RJ, Boeve BF, Tangalos JR, Petersen RC. Usefulness of MRI measures of entorhinal cortex versus hippocampus in AD. Neurology 2000; 54: 1760-1767. 